\title{
Mitochondrial fusion and fission are involved in stress tolerance of Candida glabrata
}

\author{
Shubo Li $i^{1,2,3,4}$, Liming Liu ${ }^{1,3,4^{*}}$ and Jian Chen ${ }^{1,3^{*}}$
}

\begin{abstract}
Background: Recently, cell tolerance toward environmental stresses has become the major problem in the development of industrial microbial fermentation. Acetoin is an important chemical that can be synthesized by microbes. Its toxicity was investigated using Candida glabrata as the model in this study.

Results: A series of physiological and biochemical experiments demonstrated that the organic solvent acetoin can inhibit cell growth by increasing intracellular reactive oxygen species (ROS) production and inducing damage to mitochondria and cell apoptosis. Integrating RT-PCR experiments, the genes fzo 1 and dnm 1 were overexpressed to regulate the balance between mitochondrial fusion and fission. Enhancement of mitochondrial fusion was shown to significantly increase cell tolerance toward acetoin stress by inhibiting ROS production and increasing the intracellular adenosine triphosphate (ATP) supply, which was also demonstrated by the addition of citrate.

Conclusions: Regulating mitochondrial fusion-fission may be an alternative strategy for rationally improving the growth performance of eukaryotes under high environmental stress conditions, and also expands our knowledge of the mechanisms of cell tolerance through the processes of energy-related metabolic pathways.
\end{abstract}

Keywords: Acetoin; ATP supply; Candida glabrata; Cell tolerance; Environmental stress; Mitochondrial fusion-fission

\section{Background}

With the development of metabolic engineering, microorganisms have been engineered to produce many important bulk chemicals, such as ethanol, acetone, and butanol. However, most of these are toxic to cells as they can damage cell membranes and walls and interfere with essential physiological processes [1]. Thus, improving cell tolerance has become a major challenge for microbial fermentation. To this end, genomic tools (transcriptomics, proteomics, and metabolomics) have been applied to investigate microbial responses to various solvents, and a number of strategies have been exploited to avoid solvent toxicity. Recently, a visual summary of cellular responses directed toward overcoming solvent stress and enhancing survivability was compiled [2-4], as follows: (i) metabolic detoxification, converting toxic compounds into less harmful chemicals; (ii) expression of heat shock proteins (HSP),

\footnotetext{
* Correspondence: mingll@jiangnan.edu.cn; jchen@jiangnan.edu.cn

'State Key Laboratory of Food Science and Technology, Jiangnan University,

Wuxi 214122, China

${ }^{3}$ The Key Laboratory of Industrial Biotechnology, Ministry of Education, Jiangnan University, Wuxi 214122, China

Full list of author information is available at the end of the article
}

assisting in protein folding and preventing aggregation; (iii) use of proton motive force and associated energy production; (iv) molecular efflux pumps, exporting solvents to the extracellular space; and (v) changes in cell membrane composition and biophysics, combating the fluidizing effects of solvents. But, interestingly, most of these involve energy-dependent processes, indicating that energy and energetic processes play crucial roles in protecting cells against environmental stress. For example, enhancing glucose transport and catabolism favors increased energy production and compensates for the energy expended in relieving stress in prokaryotes and eukaryotes [5-7]. Therefore, engineering the energy-generation machinery could be an alternative approach to improving cell tolerance toward environmental stress.

Mitochondria, as the power plants of the cell, can supply most of its energy through oxidative phosphorylation, and play an important role in maintaining cellular functions, such as the citric acid cycle and cell apoptosis $[8,9]$. As highly dynamic organelles, mitochondria maintain a balance between frequent cycles of fusion and fission, which tightly regulate mitochondrial number, morphology, and functions under ever-changing physiological conditions [10-12]. The 
mitochondrial fusion process can be divided into three events: docking, fusion of the outer membrane, and fusion of the inner membrane. An increase in fusion events can result in mitochondrial inner connected networks, which are protective against apoptotic sensitivity and promote survival under conditions of stress [13]. In yeast, the mechanisms of mitochondrial fusion and fission have been identified and characterized. The fusion apparatus requires the activity of the large mitochondrial GTPase mitofusin (MFN), consisting of three core components, Fzo1, Ugo1, and Mgm1, of which Fzo1 is the key component regulating lipid bilayer fusion of mitochondrial outer membranes [11]. However, for mitochondrial fission, the core apparatus needs another large GTPase, a dynamin-related protein that includes three components, Dnm1, Fis1, and Mdv1, of which Dnm1 is required for mitochondrial fission and apoptosis [12,14]. Therefore, mitochondria have the ability to coordinate a balance between fusion and fission to handle cellular and environmental stressors $[15,16]$.

Candida glabrata, a haploid multivitamin auxotrophic yeast, is an important strain used for industrial production of pyruvate [17] and $\alpha$-ketoglutaric acid [18]. Additionally, C. glabrata was also used to produce malate [19] and acetoin [20], which can serve as a high value-added platform for the food, pharmaceutical, and chemical industries as a member of the C4-dicarboxylic acid family. However, high concentrations of acetoin were found to inhibit cell growth [20], and increasing the tolerance of cells may be favorable to improving the fermentative performance of a strain. To this aim, C. glabrata was used to study the effects of acetoin stress on cellular physiological characteristics (such as reactive oxygen species (ROS) and cell viability), and the balance between mitochondrial fusion and fission was also regulated to improve cellular physiological characteristics, thus enhancing cell robustness. These results demonstrated that enhancing mitochondrial fusion to increase the adenosine triphosphate (ATP) supply may be a novel approach to improving cell tolerance toward environmental stress.

\section{Methods}

\section{Strains and plasmids}

Escherichia coli JM109 was purchased from Invitrogen (Carlsbad, CA, USA) and used for plasmid construction. $C$. glabrata $\Delta$ ura3 was derived from C. glabrata CCTCCM 202019 (multivitamin auxotroph) and used as the receptor for gene overexpression. More information about the plasmids and strains is given in Table 1.

\section{Plasmid construction and transformation}

The primers used in this work are listed in Additional file 1: Table S1, and standard cloning and bacterial transformations were performed according to Sambrook and Russell [21]. The genes $f z o 1$ and $d n m 1$ were amplified by polymerase chain reaction (PCR) from genomic DNA of $S$. cerevisiae (CEN.PK2-1C), and then inserted into the desired plasmid multi-cloning sites. In all cases, PCR was performed using TaKaRa Pyrobest DNA Polymerase (Takara Bio Inc, Shiga, Japan). All genes were sequenced to ensure correct identify of the insert prior to transformations. Yeast strains were transformed using the lithium acetate method [22].

\section{Culture medium and conditions}

During construction, strains were grown in complex (YPD) medium consisting of $10 \mathrm{~g} / \mathrm{L}$ yeast extract, $20 \mathrm{~g} / \mathrm{L}$ peptone, and $20 \mathrm{~g} / \mathrm{L}$ glucose. All engineered strains were screened on synthetic complete (SC) medium consisting of $20 \mathrm{~g} / \mathrm{L}$ glucose, $7 \mathrm{~g} / \mathrm{L}$ urea, $5 \mathrm{~g} / \mathrm{L} \mathrm{KH}_{2} \mathrm{PO}_{4}, 0.8 \mathrm{~g} / \mathrm{L}$ $\mathrm{MgSO}_{4} \cdot 7 \mathrm{H}_{2} \mathrm{O}, 3 \mathrm{~g} / \mathrm{L}$ sodium acetate, and $15 \mathrm{~g} / \mathrm{L}$ agar, at $\mathrm{pH}$ 6.0. They were fermented in a medium (medium $\mathrm{A}$ ) consisting of $100 \mathrm{~g} / \mathrm{L}$ glucose, $3 \mathrm{~g} / \mathrm{L}$ urea, $7 \mathrm{~g} / \mathrm{L} \mathrm{KH}_{2} \mathrm{PO}_{4}$, $0.8 \mathrm{~g} / \mathrm{L} \mathrm{MgSO}_{4} \cdot 7 \mathrm{H}_{2} \mathrm{O}$, and $5 \mathrm{~g} / \mathrm{L}$ sodium acetate in shake-flask culture $\left(200 \mathrm{rpm}, 30^{\circ} \mathrm{C}\right)$ using $\mathrm{CaCO}_{3}$ as the buffering agent. The inoculum size and the vitamin solution $(0.04 \mathrm{mg} / \mathrm{L}$ thiamine- $\mathrm{HCl}, 0.16 \mathrm{mg} / \mathrm{L}$ biotin, $0.4 \mathrm{mg} / \mathrm{L}$ pyridoxine- $\mathrm{HCl}$, and $8 \mathrm{mg} / \mathrm{L}$ nicotinic acid) were added to all media to constitute $15 \% v / v$ and $1 \% v / v$, respectively. When necessary, different acetoin concentrations were

Table 1 Plasmids and strains used in this study

\begin{tabular}{|c|c|c|}
\hline Strains/plasmids & Relevant characteristics & Reference \\
\hline \multicolumn{3}{|l|}{ Plasmids } \\
\hline pYX212 & $2 \mu \mathrm{m}, \mathrm{Amp}^{\mathrm{R}}, U R A 3, \mathrm{P}_{T P l}$ & Lab collection \\
\hline pYX212-fzol & Harboring the gene of fzo1 from Saccharomyces cerevisiae CEN.PK2-1C & This study \\
\hline pYX212-dnm1 & Harboring the gene of $d n m 1$ from S. cerevisiae CEN.PK2-1C & This study \\
\hline \multicolumn{3}{|l|}{ Strains } \\
\hline C. glabrata CCTCC M202019 & Multivitamin (thiamine, biotin, nicotinic acid and pyridoxine) auxotroph & [21] \\
\hline C. glabrata $\Delta$ ura3 & The mutant derived from C. glabrata CCTCC M202019 & [19] \\
\hline C-pYX & C. glabrata $\Delta$ ura3 (pYX212) & This study \\
\hline C-Fzo1 & C. glabrata $\Delta$ ura3 (pYX212- fzo1) & This study \\
\hline C-Dnm1 & C. glabrata $\Delta$ ura3 (pYX212- dnm1) & This study \\
\hline
\end{tabular}


added to the culture. Transformed E. coli JM109 cells were grown at $37^{\circ} \mathrm{C}$ in Luria-Bertani (LB) medium containing $100 \mu \mathrm{g} / \mathrm{mL}$ ampicillin.

\section{Spotting assay for evaluation of acetoin tolerance}

To evaluate microbial tolerance, a spotting assay was applied in the presence of acetoin [23]. First, the cells were incubated overnight in SC medium with shaking $\left(200 \mathrm{rpm}, 30^{\circ} \mathrm{C}\right)$, and then they were collected and resuspended by centrifugation $(10,000 \times g, 20 \mathrm{~s})$ in sterilized water. Second, the suspensions were serially diluted to an $\mathrm{OD}_{660}$ of $1 \times 10^{0}, 1 \times 10^{-1}, 1 \times 10^{-2}, 1 \times 10^{-3}, 1 \times 10^{-4}$, $1 \times 10^{-5}, 1 \times 10^{-6}, 1 \times 10^{-7}$, and $1 \times 10^{-8}$, and then spotted $(5 \mu \mathrm{L}$ each) onto agar plates containing different acetoin concentrations. The plates were sealed with vinyl plastic tape to prevent evaporation of acetoin and incubated at $30^{\circ} \mathrm{C}$.

\section{Analytical methods}

The optical absorbance at $660 \mathrm{~nm}\left(\mathrm{~A}_{660}\right)$ was converted to dry cell weight (DCW) according to the following formula [17]: $\mathrm{A}_{660} \mathrm{DCW}=1: 0.23(\mathrm{~g} / \mathrm{L})$.

\section{Cell survivability}

When the cells were incubated to $\mathrm{OD}_{660}=1.0$, some of cultures were diluted in sterile deionized water and spread evenly onto SC plates with varied acetoin concentrations $(0 \%, 10 \%$, and $15 \%)$, and the colony forming units (cfu) were determined after $24 \mathrm{~h}$ of incubation. Cell survival was expressed as survivability (cfu \%), and defined as below:

Cell survivability $=$ The number of colonies on acetoin-containing agar plate

The number of colonies on non-acetoin-containing agar plate $\times 100 \%$;

\section{Physiological parameters}

When the cells had been incubated to the exponential phase $\left(\mathrm{OD}_{660}=2.0\right)$, different acetoin concentrations were added and continual cultivation proceeded for $24 \mathrm{~h}$ $\left(200 \mathrm{rpm}, 30^{\circ} \mathrm{C}\right)$. Cells were then collected and washed three times with sterilized water. Afterwards, the OD was adjusted to 1.0 before measuring the following physiological parameters (parameters were expressed as mean values with standard error of the mean of at least three independent experiments):

(1) Mitochondrial membrane potential $\left(\Delta \Psi_{\mathrm{m}}\right): \Delta \Psi_{\mathrm{m}}$ was measured using Rh123 (Sigma, Shanghai, China) as described previously [24]. Cells were collected and washed twice with phosphate buffer saline (PBS), and the pellets were incubated with $500 \mu \mathrm{M}$ Rh123 in the dark for 10 min at room temperature. Then cells were washed with PBS three times, and the suspensions were measured using flow cytometry (FCM, BD Biosciences, Shanghai, China).

(2) ROS level: cells were collected and prepared using the ROS Assay Kit (Beyotime Institute of Biotechnology, Jiangsu, China), and ROS production was detected via flow cytometry.

(3) ATP production: cells were collected and prepared according to the protocol of the ATP Assay Kit (Beyotime Institute of Biotechnology, Jiangsu, China), and ATP levels were measured with a luminometer. Protein concentrations were determined using a BCA Protein Assay Kit (Beyotime Institute of Biotechnology, Jiangsu, China).

(4) Cell apoptosis: cell apoptosis was measured by flow cytometric analysis using the FACS Calibur (BD Biosciences, Shanghai, China). Fluorescence emission was measured through a 500/50-nm bandpass filter for Rh123-labeled cells, and through a 660/16-nm bandpass filter for PI-labeled cells. Propidium iodine (PI, Sigma, Shanghai, China) staining was used to monitor cell membrane integrity. Samples $(500 \mu \mathrm{L})$ were incubated with $3 \mu \mathrm{L}$ PI stock solution $(1 \mathrm{mg} / \mathrm{mL})$ for $5 \mathrm{~min}$ at room temperature in the dark, followed by microscopy and flow cytometry, and cell viability was calculated by measuring PI fluorescence on a log scale. Before measuring cell apoptosis and viability using staining with flow cytometry, Rh123/PI dual staining was quantified using samples from fresh cultures with or without acetoin treatment. A minimum of 10,000 events were analyzed per sample at a low flow rate. CELL QUEST software was used for data acquisition and analysis [25].

\section{Transcriptional analysis}

Total RNA isolation was carried out using an RNAprep pure Plant Kit, and reverse transcription (cDNA synthesis) was performed according to the protocol of the PrimeScript $^{\circ} R T$ reagent kit Perfect Real Time (Takara Bio Inc, Shiga, Japan). Quantitative real-time PCR (RT-PCR) was done using the $\beta$-ACTIN gene as the internal control, and the primers used in RT-PCR are given in Additional file 1: Table S1. Each sample was tested in triplicate in a 96-well plate (Bio-Rad Corp, Hercules, CA, USA).

\section{Statistical analysis}

All experiments were carried out in triplicate, and the results are expressed as mean \pm standard deviation. SPSS 18 (SPSS Statistics 18.0, SPSS Institute, Inc., Chicago, IL, 2010, USA) was used for one-way analysis of variance and canonical correlation analysis (CCA), and significant differences $(P<0.05)$ among means were determined by the least significant difference test. 


\section{Results}

\section{Tolerance of $C$. glabrata toward acetoin stress}

To determine the toxicity of the important chemical acetoin, a spotting assay on agar plates containing different acetoin concentrations was used. As shown in Figure 1a, $C$. glabrata grew on agar plates containing $6 \mathrm{~g} / \mathrm{L}$ of acetoin, but was significantly inhibited by $15 \mathrm{~g} / \mathrm{L}$ of acetoin. Furthermore, cell tolerance was also evaluated in shake-flask culture, and it was found that cell growth decreased by $7.5 \%$ (6 g/L), 19.8\% (12 g/L), 44.4\% (15 g/L), and 59.2\% (18 g/L), respectively, compared to the control conditions (without addition of acetoin) (Figure 1b). Therefore, $6 \mathrm{~g} / \mathrm{L}$ of acetoin had a slight effect on cell growth, which was consistent with the spotting assay results.

\section{Effects of acetoin stress on cellular physiological characteristics}

To investigate the toxic effects of acetoin on cells in depth, the parameters cell viability and intracellular ROS were measured under conditions of acetoin stress. Cell viability was reduced to $22 \%$ after treatment with $18 \mathrm{~g} / \mathrm{L}$ acetoin (Figure 2a), while intracellular ROS production increased to 32.5 from 14.1 compared to the control conditions (without addition of acetoin) (Figure 2b). Furthermore, double staining with Rh123 and PI, which could discriminate intact, apoptotic, and necrotic cells, was applied to observe cell apoptosis by flow cytometry. As shown in Figure 3, Rh123/PI double fluorescent staining with flow cytometry generated a contour diagram with three distinct regions: R1, necrotic cells; R2, apoptotic cells; R3, intact cells. When the concentration of acetoin increased from 0 to $18 \mathrm{~g} / \mathrm{L}$, the percentage of necrotic cells (R1) increased slightly from $0.8 \%$ to $2.15 \%$, but the percentage of apoptotic cells (R2) increased significantly to $96.48 \%$. Correspondingly, the percentage of intact cells (R3) decreased markedly from $99.22 \%$ to $1.37 \%$. Therefore, the levels of cell apoptosis were effectively increased during high levels of acetoin stress. These results demonstrate that acetoin can dramatically change the levels of cell viability and intracellular ROS, and induce cell apoptosis and death, and then significantly inhibit cell growth.

\section{Roles of mitochondrial fusion and fission in tolerance toward acetoin stress}

In yeast, mitochondria are the major source of ROS production, and the processes of mitochondrial fusion-fission are closely related to cell apoptosis [26]. Therefore, the gene expression levels of $f z o 1$ and $d n m 1$ were measured by RT-PCR under different acetoin conditions. As shown in Figure $4 \mathrm{a}$, the expression levels of $f z o 1$ and $d n m 1$ were dramatically enhanced, increased by 3.4- and 1.8-fold, respectively, at $12 \mathrm{~g} / \mathrm{L}$ of acetoin. To confirm these results, engineered C-Fzo1 and C-Dnm1 overexpressing fzo1 and $d n m 1$, respectively, were constructed using the strain CpYX (harboring the plasmid pY212) as the control. As shown in Figure 4b, no distinct differences were observed in cell growth between C-pYX, C-Fzo1, and C-Dnm1 in the absence of acetoin stress. But under high acetoin stress, the engineered strain C-pFzol exhibited superior tolerance compared to the strain C-pYX, as it grew well on an agar plate containing $18 \mathrm{~g} / \mathrm{L}$ of acetoin, and even grew in the presence of $20 \mathrm{~g} / \mathrm{L}$ acetoin. In contrast, the strain C-Dnm1 was significantly inhibited in the presence of $15 \mathrm{~g} / \mathrm{L}$ acetoin, showing lower tolerance than strain C-pYX. Therefore, increasing mitochondrial fusion improved cell tolerance, whereas increasing mitochondrial fission had a negative effect on cell tolerance toward acetoin stress.

\section{Effects of mitochondrial fusion-fission on physiological characteristics}

To elucidate the mechanisms by which mitochondrial fusion-fission regulate cell tolerance, the effects of

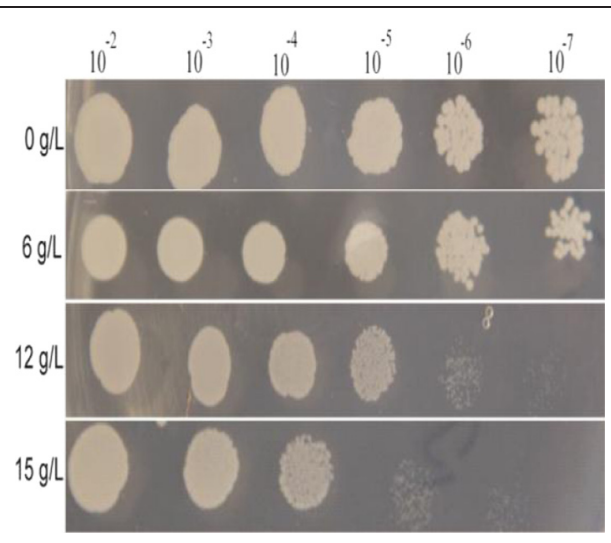

(a)

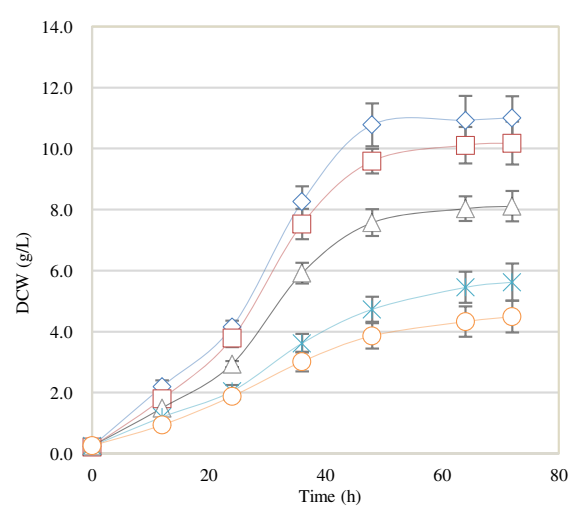

(b)

Figure 1 Cell tolerance toward acetoin stress. Application of a spotting assay (a) and time course of cell growth (DCW) in shake-flask culture with acetoin added at $0 \mathrm{~h}$ (b). Error bars represent mean values and standard deviations of three independent experiments. 


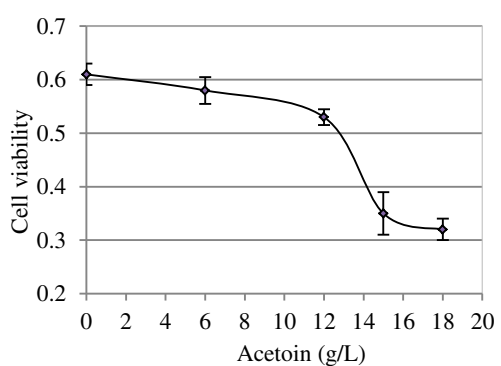

(a)

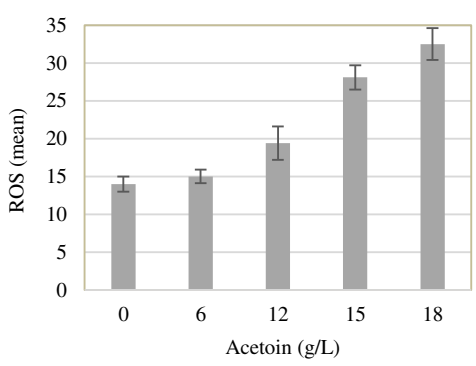

(b)

Figure 2 Effects of different acetoin concentrations on cell viability (a) and intracellular ROS production (b). Error bars represent mean values and standard deviations of three independent experiments.

mitochondrial fusion-fission on cellular physiological characteristics were further investigated. As shown in Figure 5, the intracellular environment significantly changed due to the expression of mitofusin in the engineered strains. Compared to the control conditions (without addition of acetoin), some different characteristics were observed in engineered strains cultured with $15 \mathrm{~g} / \mathrm{L}$ of acetoin, as follows. (1) Strain C-Fzo1 effectively inhibited the production of intracellular ROS (increased by only 69.3\%), which was far lower than that produced by strains C-pYX (increased by 144\%) and C-Dnm1 (increased by $180 \%$ ) (Figure $5 \mathrm{a}$ ). (2) Strain C-Fzo1 significantly maintained cell survivability and ATP production, as these parameters decreased by $44.3 \%$ and $40.7 \%$, respectively, to values that were higher than those of strains C-pYX (decreased by $64.4 \%$ and $69.7 \%$, respectively) and C-Dnm1 (decreased by $75.7 \%$ and $76.1 \%$, respectively) (Figure $5 \mathrm{~b}, \mathrm{c}$ ). Furthermore, strain C-Fzo1 more effectively maintained the balance of mitochondrial membrane potential $(\Delta \Psi \mathrm{m}$, with a decrease of $18.6 \%)$ than strain C-Dnm1 $(\triangle \Psi \mathrm{m}$ decreased by $48.2 \%)$, which may be due to lower ROS production and higher ATP formation (Figure 5d). These results demonstrate that the enhancement of mitochondrial fusion could improve the ATP supply and decrease ROS production to

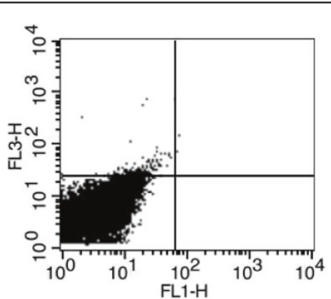

R1:0.80\%

R3:99.22\%

$(0 \mathrm{~g} / \mathrm{L})$

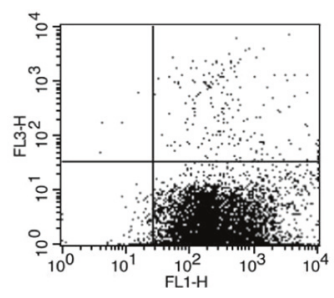

$\mathrm{R} 1: 2.15 \%$

R2:96.48\%

R3: $1.37 \%$

$(18 \mathrm{~g} / \mathrm{L})$

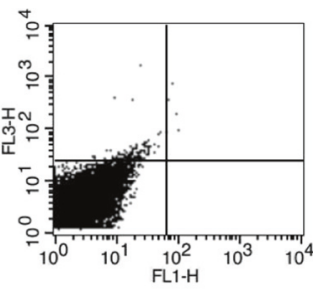

$\mathrm{R} 1: 1.10 \%$

R3:98.89\%

(6 g/L)

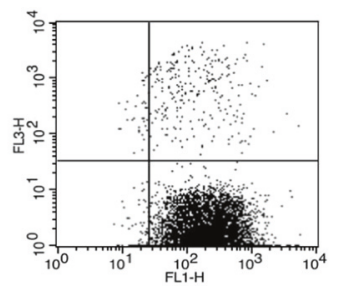

R1:2.21\%

R2:96.68\%

R3:1.08\%

(21 g/L)

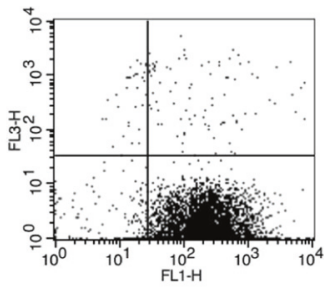

R1:4.05\%

R2:87.98\%

R3:6.97\%

(12 g/L)

Figure 3 Contour diagram of the flow cytometry of $C$. glabrata cells under the conditions of different acetoin concentrations. FL1-H, Rh123 staining; FL3-H, PI staining. Region 1 (R1), percentage of necrotic cells; region 2 (R2), percentage of apoptotic cells; region 3 (R3), percentage of intact cells. 


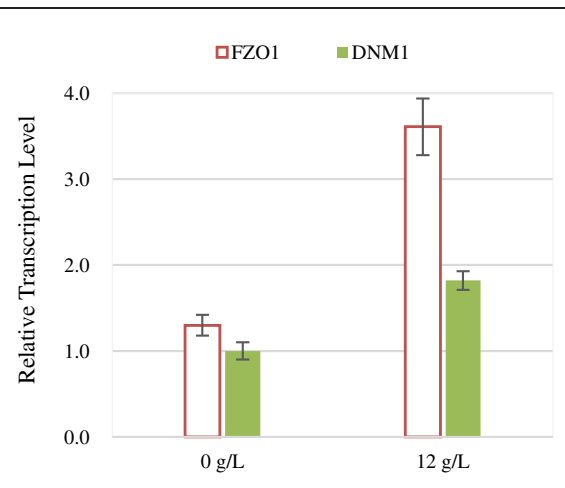

(a)

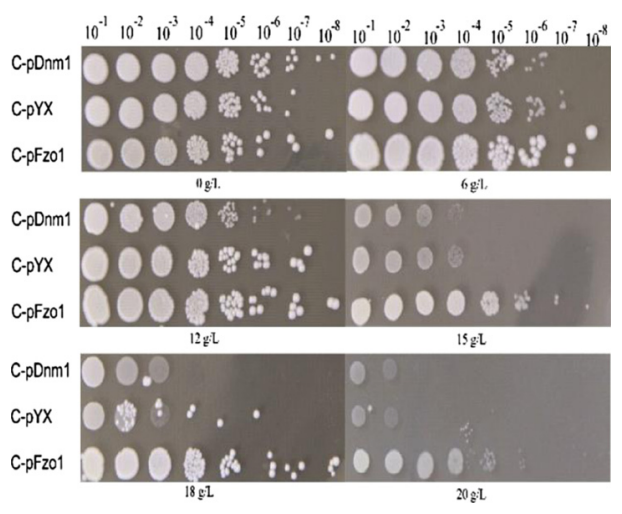

(b)

Figure 4 Roles of mitochondrial fusion-fission in cell tolerance toward acetoin stress. (a) The gene expression levels of $f z 01$ and $d n m 1$. (b) Spotting assay of the engineered strains.

maintain the balance of $\Delta \Psi \mathrm{m}$, and thus increase cell survivability and cell tolerance against acetoin stress.

\section{Effect of citrate on C. glabrata adaptation to acetoin stress} According to the results of a previous study [27], $50 \mathrm{nmol} /$ $\mathrm{L}$ of sodium citrate was added to determine its effects on cell growth and ATP production of the wild-type strain under different acetoin conditions. As shown in Figure 6a, compared to the control conditions (without addition of sodium citrate), sodium citrate effectively improved intracellular ATP supply, increasing ATP production by $18.8 \%$ (6 g/L of acetoin), 48.9\% (12 g/L of acetoin), and 11.7\% (15 g/L of acetoin). Correspondingly, cell tolerance of acetoin stress was also enhanced, so that cell growth increased by $18.2 \%$ ( $6 \mathrm{~g} / \mathrm{L}$ of acetoin), 30.1\% (12 g/L of acetoin), and $9.5 \%$ (15 g/L of acetoin) (Figure $6 \mathrm{~b}$ ). Therefore, improving the supply of cellular ATP could indeed increase cell tolerance toward acetoin stress.

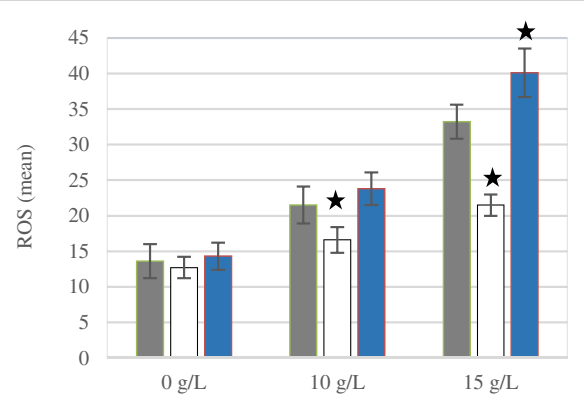

(a)

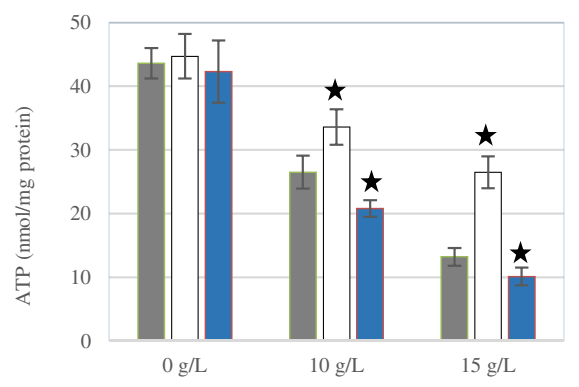

(c)

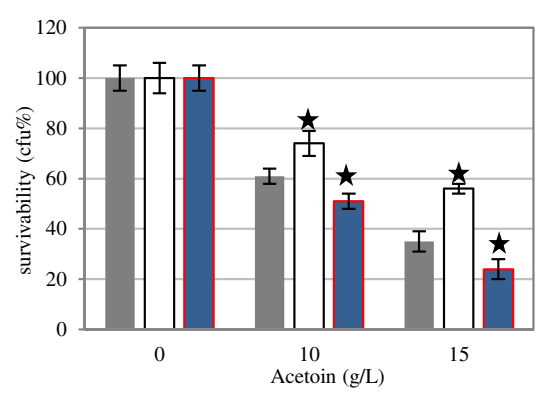

(b)

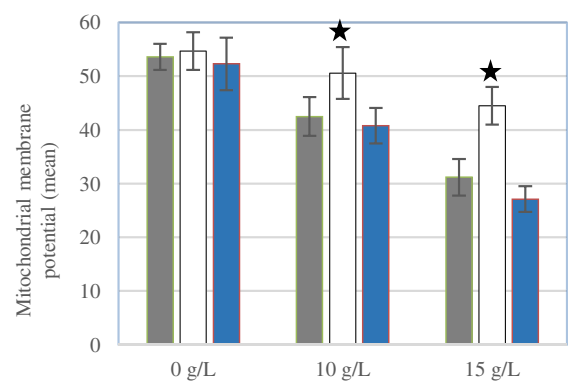

(d)

Figure 5 Effects of acetoin stress on cell physiological characteristics in the different engineered strains. (a) ROS generation. (b) Cell viability. (c) ATP production. (d) $\Delta \psi \mathrm{m}$. Error bars represent mean values and standard deviations of three independent experiments (black square, C-pYX; white square, C-Fzo1; blue square, C-Dnm1) $\left({ }^{*} P<0.05\right.$ vs. the group of strain C-pYX). 


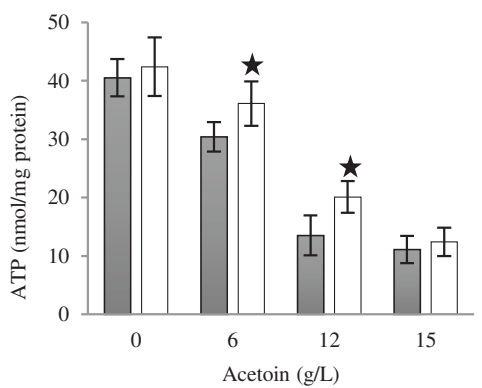

(a)

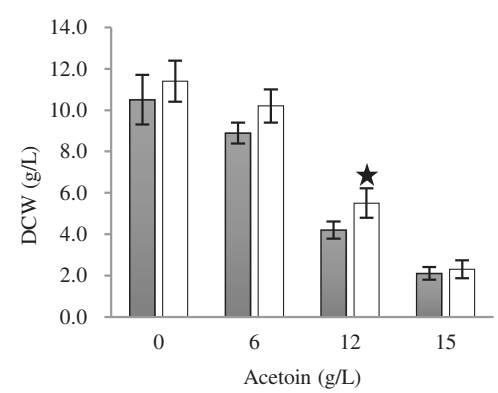

(b)

Figure 6 Effects of citrate addition on intracellular ATP production (a) and cell growth (b). (black square, without citrate addition; white square, with citrate addition). Error bars represent mean values and standard deviations of three independent experiments ${ }^{*} P<0.05$ vs. the group without citrate addition).

\section{Discussion}

In cells, mitochondria continually generate ROS as the byproduct of electron transport during oxidative phosphorylation. If ROS production increases it can damage mitochondrial components (such as proteins, lipids, and DNA) and then weaken metabolism and further increase ROS generation, causing a 'vicious downward spiral' of ROS generation and damage accumulation $[28,29]$. Additionally, intracellular ROS can participate in signaling effects to control the activity of protein kinases and phosphates and regulate gene expression, and thus induce cell apoptosis and cell death [30,31]. Here, the results indicated that acetoin stress could significantly increase intracellular ROS formation, and then decrease cell viability and induce cell apoptosis (Figures 2 and 3). Therefore, we hypothesized that reducing intracellular ROS production may be an alternative route for enhancing cell tolerance toward acetoin stress.

In eukaryotes, mitochondrial fusion-fission is important for maintaining mitochondrial numbers and morphology, and plays a critical role in sustaining functional mitochondria [32,33]. To date, the processes of mitochondrial fusion-fission have been used to investigate and explain cell death induced by different environmental stresses, such as ethanol [34], hyperosmotic [35], and acetic acid stress [36]. In such cases mitochondrial fission could facilitate apoptosis by inducing mitochondrial fragmentation and ROS production, but fusion could compensate the contents of partially damaged mitochondria and maintain energy output to mitigate various stresses. In this study, the processes of mitochondrial fusion-fission were regulated and used to enhance cellular properties to combat acetoin stress. As a result, the engineered strain C-Fzo1 overexpressing mitofusin $f z o 1$, which regulates mitochondrial fusion and maintains mitochondrial morphology, could effectively inhibit intracellular ROS formation and increase cell viability, and thus enhance cell tolerance during high levels of acetoin stress.
But, interestingly, enhancement of mitochondrial fusion could also effectively improve ATP production and help maintain the mitochondrial membrane potential $(\Delta \Psi \mathrm{m})$, which may be another reason for the high tolerance of strain C-Fzo1. Apoptosis was apparent through measurement of characteristic apoptotic indicators, such as decreased $\Delta \Psi \mathrm{m}$, production of ROS, and caspase involvement, in which $\Delta \Psi \mathrm{m}$ became transiently increased upon apoptotic stimulation in yeast cells, resulting in mitochondrial fragmentation, the release of apoptogenic factors including cytochrome $\mathrm{c}$ and apoptosis-inducing-factor (AIF) [37,38], and a permanent decrease in $\Delta \Psi \mathrm{m}$ during subsequent apoptotic processes $[39,40]$. Therefore, maintaining the mitochondrial membrane potential may be favorable for enhancing the tolerance of cells toward environmental stress. Additionally, citrate was added and this demonstrated that improving the ATP supply had a positive effect on cell growth in the presence of acetoin stress. Actually, mitochondrial fission-fusion has been shown to alter energy requirements to regulate cell components, so that hyperfused mitochondria led to an increase in mitochondrial oxygen consumption and ATP formation that enhanced cell growth [41,42]. Therefore, the results reported here provide an alternative strategy for rationally improving the growth performance of eukaryotes, especially those that are organic solvent producers, under high organic solvent conditions, and also expand our knowledge of the mechanism of organic solvent tolerance through the processes of energy-related metabolic pathways.

\section{Conclusions}

In this study, the processes of mitochondrial fusion-fission were regulated and used to inhibit intracellular ROS production, raise the intracellular ATP supply, and maintain the mitochondrial membrane potential, and therefore increase cell tolerance toward acetoin stress. However, mitochondrial fission-fusion can also regulate many other 
physiological functions, such as $\mathrm{Ca}^{2+}$ homeostasis [43], metabolite transportation, and signaling exchange [32], to maintain the metabolic stability of mitochondria. Hence, the roles of mitochondrial fission-fusion in influencing the intracellular environment and physiological characteristics require further comprehensive investigation, exploiting some novel strategies to improve the performance of industrial microorganisms in a future study.

\section{Additional file}

Additional file 1: Table S1. Primers used for RT-PCR and PCR amplification in this study.

\section{Competing interests}

The authors declare that they have no competing interests.

\section{Authors' contributions}

SBL and LML conceived the study. SBL made contributions to the design of the experiments, the acquisition of data, the analysis and interpretation of data and contributed to the manuscript writing. LML and JC conceived and organized the study and helped to draft the manuscript, and have revised the manuscript. All authors read and approved the final manuscript.

\section{Acknowledgements}

This research was financially supported by the Major State Basic Research Development Program of China (973 Program, No. 2013CB733602), the Program for Young Talents in China, the Provincial Outstanding Youth Foundation of Jiangsu Province (BK2012002), the Priority Academic Program Development of Jiangsu Higher Education Institutions, the National Natural Science Foundation of China (31270079) and the Fund from Wuxi City (CLE01N1111).

\section{Author details \\ 'State Key Laboratory of Food Science and Technology, Jiangnan University, Wuxi 214122, China. ${ }^{2}$ College of Light Industry and Food Engineering, Guangxi University, Nanning 530004, China. ${ }^{3}$ The Key Laboratory of Industrial Biotechnology, Ministry of Education, Jiangnan University, Wuxi 214122, China. ${ }^{4}$ Laboratory of Food Microbial-Manufacturing Engineering, Jiangnan University, Wuxi 214122, China.}

Received: 26 October 2014 Accepted: 17 February 2015

Published online: 12 March 2015

\section{References}

1. Dunlop MJ (2011) Engineering microbes for tolerance to next-generation biofuels. Biotechnology for Biofuels 4:32

2. Ramos JL, Duque E, Gallegos MT, Godoy P, Ramos-Gonzalez MI, Rojas A, et al. (2002) Mechanisms of solvent tolerance in gram-negative bacteria. Annual Review of Microbiology 56:743-768

3. Ma MG, Liu ZL (2010) Mechanisms of ethanol tolerance in Saccharomyces cerevisiae. Appl Microbiol Biot 87(3):829-845

4. Nicolaou SA, Gaida SM, Papoutsakis ET (2010) A comparative view of metabolite and substrate stress and tolerance in microbial bioprocessing: from biofuels and chemicals, to biocatalysis and bioremediation. Metabolic Engineering 12(4):307-331

5. Segura A, Godoy P, van Dillewijn P, Hurtado A, Arroyo N, Santacruz S, et al. (2005) Proteomic analysis reveals the participation of energy- and stress-related proteins in the response of Pseudomonas putida DOT-T1E to toluene. Journal of Bacteriology 187(17):5937-5945

6. Piper PW (1995) The heat-shock and ethanol stress responses of yeast exhibit extensive similarity and functional overlap. FEMS Microbiology Letters 134(2-3):121-127

7. Alsaker KV, Paredes C, Papoutsakis ET (2010) Metabolite stress and tolerance in the production of biofuels and chemicals: gene-expression-based systems analysis of butanol, butyrate, and acetate stresses in the anaerobe Clostridium acetobutylicum. Biotechnology and Bioengineering 105(6):1131-1147
8. Green DR, Reed JC (1998) Mitochondria and apoptosis. Science 281(5381):1309-1312

9. Chan DC (2006) Mitochondria: dynamic organelles in disease, aging, and development. Cell 125(7):1241-1252

10. Detmer SA, Chan DC (2007) Functions and dysfunctions of mitochondrial dynamics. Nature Reviews. Molecular Cell Biology 8(11):870-879

11. Westermann B (2008) Molecular machinery of mitochondrial fusion and fission. Journal of Biological Chemistry 283(20):13501-13505

12. Westermann $B$ (2010) Mitochondrial dynamics in model organisms: what yeasts, worms and flies have taught us about fusion and fission of mitochondria. Seminars in Cell \& Developmental Biology 21(6):542-549

13. Chen HC, McCaffery JM, Chan DC (2007) Mitochondrial fusion protects against neurodegeneration in the cerebellum. Cell 130(3):548-562

14. Rapaport D, Brunner M, Neupert W, Westermann B (1998) Fzo1p is a mitochondrial outer membrane protein essential for the biogenesis of functional mitochondria in Saccharomyces cerevisiae. Journal of Biological Chemistry 273(32):20150-20155

15. Youle RJ, Karbowski M (2005) Mitochondrial fission in apoptosis. Nature Reviews. Molecular Cell Biology 6(8):657-663

16. Santel A, Frank S (2008) Shaping mitochondria: the complex posttranslational regulation of the mitochondrial fission protein DRP1. IUBMB Life 60(7):448-455

17. Liu LM, Li Y, Li HZ, Chen J (2004) Manipulating the pyruvate dehydrogenase bypass of a multi-vitamin auxotrophic yeast Torulopsis glabrata enhanced pyruvate production. Letters in Applied Microbiology 39(2):199-206

18. Xu N, Liu LM, Zou W, Liu J, Hua Q, Chen J (2013) Reconstruction and analysis of the genome-scale metabolic network of Candida glabrata. Molecular BioSystems 9(2):205-216

19. Chen XL, Xu GQ, Xu N, Zou W, Zhu P, Liu LM, et al. (2013) Metabolic engineering of Torulopsis glabrata for malate production. Metabolic Engineering 19:10-16

20. Li SB, Xu N, Liu LM, Chen J (2014) Engineering of carboligase activity reaction in Candida glabrata for acetoin production. Metabolic Engineering 22:32-39

21. Maniatis T, Fritsch EF, Sambrook J (1982) Molecular cloning: a laboratory manual. vol. 545. Cold Spring Harbor Laboratory, Cold Spring Harbor, NY

22. Zhou JW, Dong ZY, Liu LM, Du GC, Chen J (2009) A reusable method for construction of non-marker large fragment deletion yeast auxotroph strains: a practice in Torulopsis glabrata. Journal of Microbiological Methods 76(1):70-74

23. Nishida N, Ozato N, Matsui K, Kuroda K, Ueda M (2013) ABC transporters and cell wall proteins involved in organic solvent tolerance in Saccharomyces cerevisiae. Journal of Biotechnology 165(2):145-152

24. Ludovico P, Sansonetty F, Corte-Real M (2001) Assessment of mitochondrial membrane potential in yeast cell populations by flow cytometry. Microbiology-Sgm 147(12):3335-3343

25. Sharon LM, Thomas GC (1999) Inhibition of caspase activity delays apoptosis in a transfected NS/0 myeloma cell line. Biotechnology and Bioengineering 67(2):165-176

26. Breitenbach M, Laun P, Gimona M (2005) The actin cytoskeleton, RAS-cAMP signaling and mitochondrial ROS in yeast apoptosis. Trends in Cell Biology 15(12):637-639

27. Zhou J, Liu L, Chen J (2011) Improved ATP supply enhances acid tolerance of Candida glabrata during pyruvic acid production. Journal of Applied Microbiology 110(1):44-53

28. Andreyev AY, Kushnareva YE, Starkov AA (2005) Mitochondrial metabolism of reactive oxygen species. Biochemistry Biokhimiia 70(2):200-214

29. Sedensky MM, Morgan PG (2006) Mitochondrial respiration and reactive oxygen species in C. elegans. Experimental Gerontology 41(10):957-967

30. Allen RG, Tresini M (2000) Oxidative stress and gene regulation. Free Radical Bio Med 28(3):463-499

31. Ischiropoulos H, Beckman JS (2003) Oxidative stress and nitration in neurodegeneration: cause, effect, or association? Journal of Clinical Investigation 111(2):163-169

32. Otera $\mathrm{H}$, Ishihara N, Mihara K (2013) New insights into the function and regulation of mitochondrial fission. Bba-Mol Cell Res 1833(5):1256-1268

33. Youle RJ, van der Bliek AM (2012) Mitochondrial fission, fusion, and stress. Science 337(6098):1062-1065

34. Kitagaki H, Araki Y, Funato K, Shimoi H (2007) Ethanol-induced death in yeast exhibits features of apoptosis mediated by mitochondrial fission pathway. FEBS Letters 581(16):2935-2942

35. Criollo A, Galluzzi L, Maiuri MC, Tasdemir E, Lavandero S, Kroemer G (2007) Mitochondrial control of cell death induced by hyperosmotic stress. Apoptosis 12(1):3-18 
36. Ludovico P, Rodrigues F, Almeida A, Silva MT, Barrientos A, Corte-Real M (2002) Cytochrome $\mathrm{c}$ release and mitochondria involvement in programmed cell death induced by acetic acid in Saccharomyces cerevisiae. Molecular Biology of the Cell 13(8):2598-2606

37. Kroemer G, Reed JC (2000) Mitochondrial control of cell death. Nature Medicine 6(5):513-519

38. Creagh EM, Martin SJ (2001) Caspases: cellular demolition experts. Biochemical Society Transactions 29(6):696-702

39. Pozniakovsky Al, Knorre DA, Markova OV, Hyman AA, Skulachev VP, Severin FF (2005) Role of mitochondria in the pheromone- and amiodaroneinduced programmed death of yeast. Journal of Cell Biology 168(2):257-269

40. Yu XX, Wang HJ, Liu LM (2014) Two non-exclusive strategies employed to protect Torulopsis glabrata against hyperosmotic stress. Appl Microbiol Biot 98(7):3099-3110

41. Mitra K, Wunder C, Roysam B, Lin G, Lippincott-Schwartz J (2009) A hyperfused mitochondrial state achieved at $\mathrm{G}(1)-S$ regulates cyclin $E$ buildup and entry into $S$ phase. Proceedings of the National Academy of Sciences of the United States of America 106(29):11960-11965

42. Mitra K (2013) Mitochondrial fission-fusion as an emerging key regulator of cell proliferation and differentiation. BioEssays 35(11):955-964

43. Jezek P, Plecita-Hlavata L (2009) Mitochondrial reticulum network dynamics in relation to oxidative stress, redox regulation, and hypoxia. International Journal of Biochemistry \& Cell Biology 41(10):1790-1804

\section{Submit your manuscript to a SpringerOpen ${ }^{\circ}$ journal and benefit from:}

- Convenient online submission

- Rigorous peer review

- Immediate publication on acceptance

- Open access: articles freely available online

- High visibility within the field

- Retaining the copyright to your article

Submit your next manuscript at $>$ springeropen.com 